CASE REPORT

F.A. Marden

C.M. Putman

\section{Cement-Embolic Stroke Associated with Vertebroplasty}

SUMMARY: Major neurologic morbidity and mortality with vertebroplasty are rare. We describe a 71-year-old woman who had a stroke approximately 30 minutes after vertebroplasty. Imaging revealed that bone cement had embolized to the left middle cerebral artery. Emergency embolectomy was attempted but was unsuccessful. This report emphasizes the need to be aware that intracranial embolization of polymethylmethacrylate can occur and describes management strategies to consider.
V ertebroplasty is considered a very safe and effective procedure to treat painful compression fractures, with nearly $90 \%$ of patients experiencing some pain relief. ${ }^{1}$ Symptomatic complications are uncommon, reported to occur in the range of $1 \%-3 \%$ for osteoporotic compression fractures and slightly more for neoplastic ones. The most feared complications are related to extravertebral extrusion of cement. This has been described in approximately $41 \%$ of patients; fortunately, it rarely leads to symptoms. ${ }^{1}$ A small amount of polymethylmethacrylate (PMMA) leakage into the paravertebral soft tissues or intervertebral disk space is generally of no clinical significance. Even pulmonary cement embolism from leakage into the paravertebral venous plexus, which has been reported to occur in up to $5 \%$ of patients, is typically well tolerated. ${ }^{2}$ Major neurologic injury may occur as a result of spinal cord or nerve root compression from cement extrusion into the spinal canal or neuroforamen. ${ }^{3}$ Reports of procedure-related death are rare; causes have been attributed to anaphylactic reactions to the PMMA, ${ }^{3}$ pulmonary cement emboli, ${ }^{4,5}$ and fat emboli. ${ }^{6}$ We report an unusual case of intracranial embolization of PMMA with vertebroplasty and discuss possible management options.

\section{Case Report}

A 71-year-old woman presented with severe back pain due to a recent T12 compression fracture. She underwent percutaneous vertebroplasty in standard fashion via a bilateral transpedicular approach (SpinePlex Bone Cement, 1/2 dose; Stryker Instruments, Kalamazoo, Mich). There was filling of the vertebral body with only a small amount of leakage into the prevertebral space and paraspinal veins in amounts typically of no clinical significance (Fig 1).

Approximately 30 minutes later in the recovery room, the patient suddenly became severely aphasic with a right hemiparesis (National Institutes of Health Stroke Scale score, 20). CT/CT angiography (CTA) of the head and intracranial circulation revealed a small round focal radiodensity along the left middle cerebral artery (MCA), measuring approximately $1500 \mathrm{HU}$, consistent with an embolus of PMMA (Fig 2).

The patient was subsequently brought to the angiography suite for attempted removal of the embolus. Several small scattered PMMA emboli were seen within the lungs on fluoroscopy. Cerebral angiog-

Received March 27, 2008; accepted April 7

From the Department of Radiology (F.A.M.), Inova Fairfax Hospital, Falls Church, Va; and Department of Neurosurgery (C.M.P.), George Washington University, Washington, DC.

Please address correspondence to F. Marden, MD, Department of Interventional Neuroradiology, Alexian Brothers Medical Center, 800 Biesterfield Rd, Eberle Bldg, Suite 610, Elk Grove Village, IL 60007; e-mail: mardenf@alexian.net

DOl 10.3174/ajnr.A1159 raphy revealed a similar-appearing embolus in the left MCA, associated with a more distal occlusion of the vessel (Fig 3).

The patient was systemically anticoagulated with heparin to help prevent thrombus propagation. Numerous attempts subsequently to extract the embolus were unsuccessful, using the Merci X6 retriever (Concentric Medical, Mountain View, Calif), followed by Alligator 3.0- and 4.0-mm Retrieval Devices (Chestnut Medical Technologies, Menlo Park, Calif). The Merci device was able to retrieve a small amount of thrombus. Unfortunately, none of the devices were able to snare the embolus fully. Therefore, to optimize distal perfusion, we pushed the cement embolus into the origin of the superior M2 branch at the MCA trifurcation by using the Merci 14X microcatheter (Fig 4).

Open surgical embolectomy was considered, but because of the patient's poor general medical condition, her family declined further aggressive measures. The patient died within 24 hours as a result of her stroke.

\section{Discussion}

Our patient developed an ischemic stroke approximately 30 minutes after undergoing vertebroplasty, and imaging showed that a small amount of PMMA had embolized to the brain. Because further testing and autopsy were declined by the family, the underlying etiology remains uncertain. However, with the unexpected presence of a small amount of PMMA in the lungs detected after the vertebroplasty, we suspect that the patient had a right-to-left shunt from either a patent foramen ovale or a pulmonary arteriovenous malformation, which allowed intracranial embolization to occur unrecognized during the procedure. The reason for the delay in symptoms, then, may seem counterintuitive, because PMMA typically solidifies within minutes and does not subsequently migrate even if it has extravasated into a vessel. We hypothesize that initially the PMMA was only partially obstructive, because the native image taken during angiography showed that the embolus had an open center and the initial CTA and angiographic images showed contrast dye beyond the PMMA embolus and it was not until 30 minutes later when complete thrombosis of the vessel occurred that the patient became symptomatic.

Treatment options are limited for this uncommon scenario. Endovascular embolectomy was immediately attempted by using both the Merci and Alligator devices, which have been approved by the US Food and Drug Administration for retrieval of foreign bodies in the peripheral vasculature and neurovasculature. Unfortunately, the loops of the Merci X6 device could not fully entrap the embolus and, upon withdrawal, would unravel. It is possible that the newer Merci L5 or L6 devices, not available at 

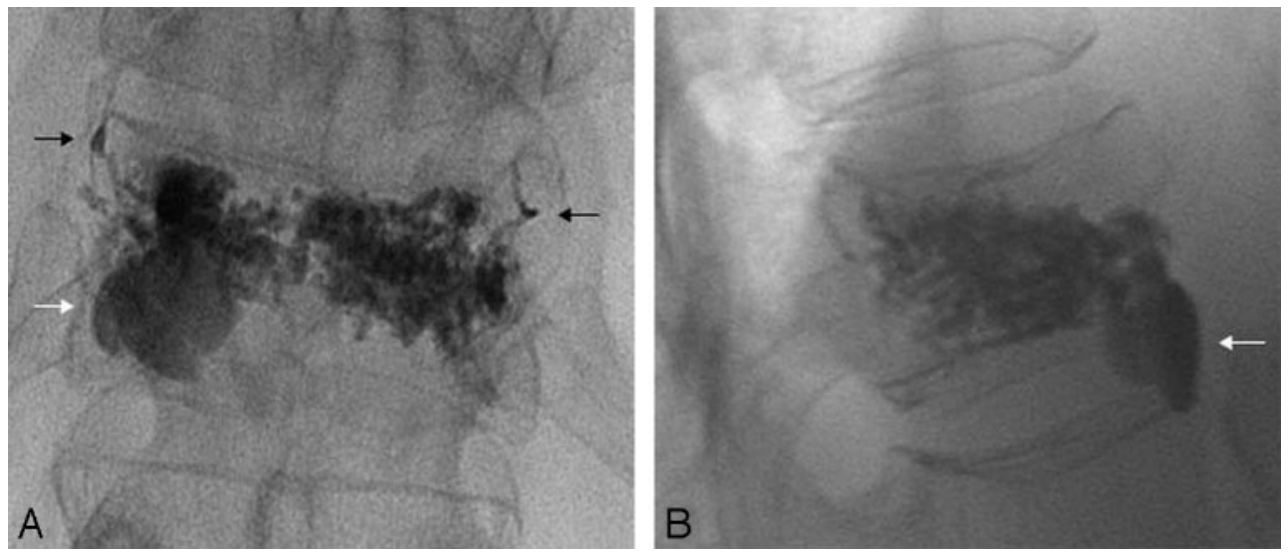

Fig 1. Fluoroscopic images from the vertebroplasty procedure show a mild spinal compression fracture and PMMA filling the vertebra ( $A$, posterior-anterior; $B$, lateral). There is a small amount of extrusion into the prevertebral soft tissues on the left (white arrow) and paraspinal veins (black arrows).
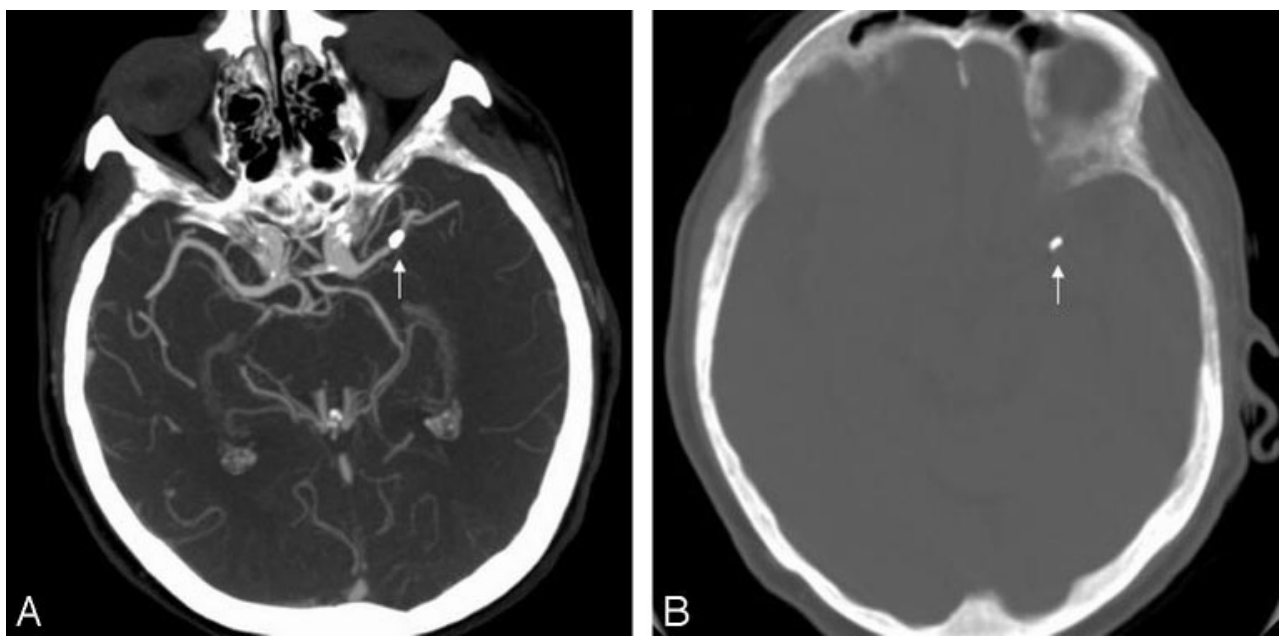

Fig 2. CTA shows a round focal density in the M1 segment of the left MCA (arrow), consistent with PMMA ( $A$, maximum intensity projection image; $B$, bone window).
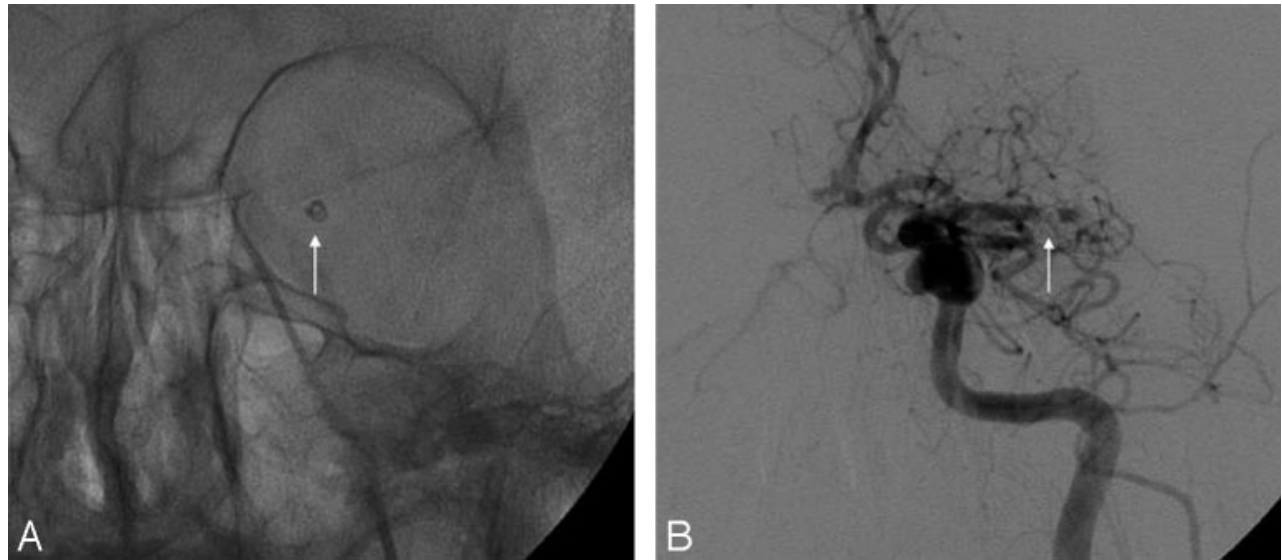

Fig 3. Angiography shows a small round embolus with the same radio-opacity as PMMA in the proximal left MCA (arrow), associated with an adjacent arterial occlusion (A, native image; $B$, digital subtraction angiography).

the time of this procedure, would have enabled retrieval. The Alligator device was also unsuccessful, because the jaws opened at too small an angle to allow the distal hooks to grasp the embolus. Thrombolytic therapy to treat any associated bland thrombus may have been an option at this time, though the MCA may well have reoccluded without removal of the underlying foreign body. Instead in this pa- tient, because the embolus could not be withdrawn, it was hoped that distal perfusion and collateral flow could be maximized by advancing the embolus into a distal branch. This proved to be relatively easy by using the Merci microcatheter. At that point, open surgical embolectomy was discussed, because it has been successful in selective ischemic stroke cases due to thromboemboli and other foreign bod- 


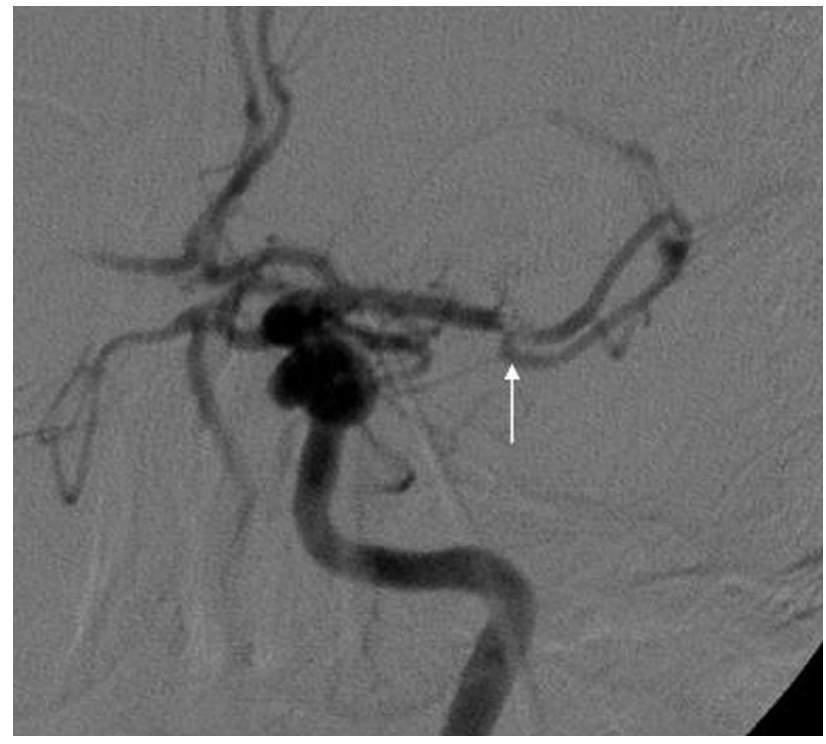

Fig 4. Angiography after the embolus had been advanced distally to the MCA trifurcation (arrow). Flow is restored in the middle and inferior M2 branches. However, the superior M2 branch is occluded.

ies that have embolized intracranially. ${ }^{7,8}$ However, the family declined.

\section{Conclusions}

We describe a case of fatal intracranial embolization of PMMA with vertebroplasty. Although this procedure is considered very safe and small amounts of extrusion of cement into the soft tissues and paravertebral veins are typically well tolerated, this report emphasizes the need to be aware that paradoxical embolization can occur and can paradoxically become symptomatic in delayed fashion. Management options to consider in the acute setting may include embolectomy by using an endovascular retrieval device, thrombolytic therapy for any associated thrombus, and possibly open surgical embolectomy.

\section{References}

1. Hulme PA, Krebs J, Ferguson SJ, et al. Vertebroplasty and kyphoplasty: a systematic review of 69 clinical studies. Spine 2006;31:1983-2001

2. Choe DH, Marom EM, Ahrar K, et al. Pulmonary embolism of polymethyl methacrylate during percutaneous vertebroplasty and kyphoplasty. AJR Am J Roentgenol 2004;183:1097-102

3. Nussbaum DA, Gailloud P, Murphy K. A review of complications associated with vertebroplasty and kyphoplasty as reported to the Food and Drug Administration medical device related web site. J Vasc Interv Radiol 2004;15: 1185-92

4. Monticelli F, Meyer HJ, Tutsch-Bauer E. Fatal pulmonary cement embolism following percutaneous vertebroplasty (PVP). Forensic Sci Int 2005; 149:35-38

5. Yoo KY, Jeong SW, Yoon W, et al. Acute respiratory distress syndrome associated with pulmonary cement embolism following percutaneous vertebroplasty with polymethylmethacrylate. Spine 2004;29:E294-97

6. Syed MI, Jan S, Patel NA, et al. Fatal fat embolism after vertebroplasty: identification of the high-risk patient. AJNR Am J Neuroradiol 2006;27:343-45

7. Horiuchi T, Nitta J, Sakai K, et al. Emergency embolectomy for treatment of acute middle cerebral artery occlusion. J Neurosurg 2007;106:257-62

8. Haninec P, Houstava L, Klener J. Shotgun pellet embolus of the middle cerebral artery treated by emergency embolectomy. Br J Neurosurg 1996; $10: 311-14$ 